\title{
ON THE CENTRAL LIMIT THEOREM IN $R_{k}$
}

\author{
BY R. RANGA RAO
}

Communicated by J. L. Doob, March 6, 1961

Let $\xi_{1}, \xi_{2}, \cdots, \xi_{n}, \cdots$, be a sequence of independent and identically distributed random vectors in $R_{k}$ with finite second order moments. Let $\eta_{n}=\left(\xi_{1}+\cdots+\xi_{n}\right) n^{-1 / 2}$ and let $P_{n}(A)=P\left[\eta_{n} \in A\right]$. Let $\eta$ denote a random vector in $R_{k}$ which is normally distributed and whose moments of the first two orders are identical with those of $\xi_{1}$ and let $P(A)=P[\eta \in A]$. Then, by the central limit theorem in $R_{k}, P_{n}$ weakly converges to $P$. A question that arises naturally here is an investigation of the error of approximation $P_{n}-P$. This problem has been thoroughly investigated in the case $k=1$ (cf. $[3 ; 4 ; 5]$ and also the survey [6] where a complete set of references is given). For $k>1$, Bergström $[1 ; 2]$ obtained bounds on the error

$$
\sup _{x \in R_{k}}\left|F_{n}(x)-\Phi(x)\right|
$$

where $F_{n}, \Phi$ are the distribution functions of $\eta_{n}$ and $\eta$ respectively. Esseen [5] gave similar bounds for the error $\left|P_{n}(A)-P(A)\right|$, when $A$ is a sphere with centre at the origin. The object of this study is to investigate the error $\Delta_{n}(A)=P_{n}(A)-P(A)$ for a very wide class of sets-namely the class of all convex subsets of $R_{k}$.

2. Notation and preliminaries. Let $\xi_{1}=\left(\xi_{1}^{(1)}, \ldots, \xi_{1}^{(k)}\right)$. We suppose that $E \xi_{1}^{(j)}=0$ for $j=1,2, \cdots, k$, and that the variance covariance matrix of $\xi_{1}$, to be denoted by $V$, is nonsingular. We use the following notation for denoting the moments and cumulants of $\xi_{1}$ :

$$
\beta_{s}=\sum_{j=1}^{k} E\left|\xi_{1}^{(j)}\right|^{8}
$$

the cumulant of order $\left(s_{1}, s_{2}, \cdots, s_{k}\right)$ will be denoted by $\lambda_{1}^{s_{1}} \cdot \lambda_{2}^{s_{2}} \ldots$ $\cdot \lambda_{k}^{s_{k}}$. Let $f(t)$ denote the characteristic function of $\xi_{1}$. Then the characteristic function of $\eta_{n}$ is $\left[f\left(t n^{-1 / 2}\right)\right]^{n}$. Let the polynomials $\widetilde{P}_{j}(w)$ in the vector $w=\left(w_{1}, \cdots, w_{k}\right)$ be defined by the formal identity:

$$
\exp \left\{\sum_{j=3}^{\infty} \frac{1}{j !}\left(\lambda_{1} w_{1}+\cdots+\lambda_{k} w_{k}\right)^{i} n^{-(j-2) / 2}\right\}=\sum_{j=0}^{\infty} n^{-j / 2} \widetilde{P}_{j}(w) .
$$

(Here the $\lambda$ 's represent the cumulants of $\xi_{1}$.) Let the functions $\widetilde{P}_{j}(-\phi), \widetilde{P}_{j}(-\Phi)$ for $j=0,1, \cdots$, be defined as follows: 


$$
\tilde{P}_{j}(-\phi)(x)=(2 \pi)^{-k} \int \tilde{P}_{j}(i t) \exp \left[-i t^{\prime} \cdot x-\frac{1}{2} t^{\prime} V^{-1} t\right] d t
$$

and

$$
\tilde{P}_{j}(-\Phi)(x)=\int_{\left[y_{j} \leqq x_{j} ; j=1,2, \cdots, k\right]} \tilde{P}_{j}(-\phi)(y) d y_{1} \cdots d y_{k} .
$$

A random vector $\xi$ in $R_{k}$ is said to be a lattice vector if there is a lattice $\mathscr{L}$ of points in $R_{k}$ such that $P[\xi \in \mathfrak{L}]=1$. The lattice $\mathfrak{L}$ is said to be minimal if there is no proper sublattice $\mathfrak{L}_{1}$ of $\mathscr{L}$ such that $P\left[\xi \in \mathscr{L}_{1}\right]=1$. If $\xi_{1}$ is a lattice vector we may always suppose without loss of generality that the minimal lattice in which $\xi_{1}$ is concentrated is $\mathfrak{L}_{0}=[a+m$; where $a$ is some fixed vector and $m$ is an arbitrary vector such that $m=\left(m_{1}, \cdots, m_{k}\right)$ where each $m_{j}$ is an integer positive, negative or zero]. If $\xi_{1}$ is a lattice vector then we define

$$
p_{n}(z)=P\left[\xi_{1}+\cdots+\xi_{n}=z\right]=P\left[\eta_{n}=z n^{-1 / 2}\right] .
$$

\section{Theorems.}

THEOREM 1. Suppose that $\beta_{4}<\infty$ and that the variance covariance matrix of $\xi_{1}$ is the identity matrix. Then

$$
\left|P_{n}(A)-P(A)\right| \leqq c(k) \beta_{4}^{3 / 2}(\log n)^{\alpha} n^{-1 / 2}
$$

uniformly for all (measurable) convex subsets $A$ of $R_{k}$. In (5), $\alpha=(k-1) / 2(k+1)$ and $c(k)$ is a constant depending only on $k$.

Theorem 2. Suppose that the characteristic function of $\xi_{1}$ satisfies the condition (C):

$$
\limsup _{|t| \rightarrow \infty}|f(t)|<1
$$

If $\beta_{8}<\infty \quad(s \geqq 3)$ then

$$
P_{n}(A)=\sum_{j=0}^{8-3} n^{-j / 2} \int_{A} d \widetilde{P}_{j}(-\Phi)+O\left\{(\log n)^{(k-1) / 2} n^{-(8-2) / 2}\right\}
$$

uniformly for all (measurable) convex subsets of $R_{k}$, where the functions $\tilde{P}_{j}(-\Phi)$ are defined by (3).

Now suppose that $\xi_{1}$ is a lattice vector concentrated in the minimal lattice $\mathfrak{L}_{0}$. Let $S_{1}(u)=u-[u]+1 / 2$ for all real numbers $u$. Then we have

THEOREM 3. If $\beta_{8}<\infty$, then 


$$
\sum_{z \in \mathfrak{L}_{0}}\left|p_{n}(z)-q_{n}^{(s)}(z)\right|=O\left\{(\log n)^{k / 2} n^{-(8-2) / 2}\right\}
$$

where $p_{n}(z)$ is defined by (4) and

$$
q_{n}^{(s)}(z)=\sum_{j=0}^{s-3} n^{-j / 2} \tilde{P}_{j}(-\phi)(z) .
$$

Theorem 4. Suppose $\beta_{5}<\infty$. Then

$$
P_{n}(A)=\int_{A} d Q(x)+n^{-1 / 2} \int_{A} d \tilde{P}_{1}(-\Phi)+O\left(n^{-1}\right)
$$

uniformly for all Borel sets $A$, where

$$
Q(x)=\prod_{j=1}^{k}\left[1-n^{-1 / 2} S_{1}\left(x_{j} n^{1 / 2}-n a_{j}\right) \frac{\partial}{\partial x_{j}}\right] \Phi(x) .
$$

In particular

$F_{n}(x)=\Phi(x)+n^{-1 / 2} \widetilde{P}_{1}(-\Phi)-n^{-1 / 2} \sum_{j=1}^{k} S_{1}\left(x_{j} n^{1 / 2}-n a_{j}\right) \frac{\partial \Phi}{\partial x_{j}}+O\left(n^{-1}\right)$

uniformly for $x$ in $R_{k}$.

For Theorems 1 and 2 the method followed is a convolution method similar to the one employed by Esseen [5]. Theorem 3 is easily proved by standard techniques of Fourier analysis, and the transition from Theorem 3 to Theorem 4 is effected through a generalization of the classical Euler-Maclaurin sum formula to functions of several variables. The details of proofs will appear elsewhere.

The author is greatly indebted to R. R. Bahadur for encouragement and for valuable suggestions and discussions.

\section{REFERENCES}

1. H. Bergström, On the central limit theorem in $R_{k}, k>1$, Skand. Aktuarietidskr. vol. 28 (1945) pp. 106-127.

2. - On the central limit theorem in the case of not equallydistributed random variables, Skand. Aktuarietidskr. vol. 33 (1949) pp. 37-62.

3. A. C. Berry, On the accuracy of Gaussian approximation to the sum of independent variates, Trans. Amer. Math. Soc. vol. 49 (1941) pp. 122-136.

4. H. Cramér, Random variables and probability distributions, Cambridge Tracts, No. 36, 1937.

5. C. G. Esseen, Fourier analysis of distribution functions. A mathematical study of the Laplace-Gaussian law, Acta. Math. vol. 77 (1945) pp. 1-125.

6. D. L. Wallace, Asymptotic approximations to distributions, Ann. Math. Statist. vol. 29 (1958) pp. 635-654.

Indian Statistical Institute, Calcutta 